\title{
Stabilität von Bewertungen zur Auswahl von Systemlösungen am Beispiel automatisierter Kommissioniersysteme für Beutelware
}

\author{
DR.-ING. THORSTEN SCHMIDT \\ M.S.I.E. (USA) LARS LEIKING \\ FRAUNHOFER-INSTITUT FÜR MATERIALFLUSS UND LOGISTIK IML, DORTMUND
}

\begin{abstract}
Der Druck auf Kosteneinsparungen und Produktivitätszuwachs in Bereichen der Kommissionierung und somit auch der Wunsch nach Rationalisierung durch Automatisierung wachsen stetig. Besonders bei in Beuteln verpackten Artikeln gestaltet sich jedoch eine Automatisierung der Kommissioniervorgänge aufgrund der mechanischen Produkteigenschaften schwierig. Entsprechende Systeme sind erst noch zu entwickeln. Im Systementwicklungsprozess möchte man möglichst frühzeitig Entscheidungen treffen können, um die technisch-wirtschaftlich sinnvollste Lösungsvariante auszuwählen und Entwicklungsaufwand einzusparen. Dieser Artikel beschäftigt sich mit den Eigenschaften besagter Produkte im Hinblick auf deren automatisierte Handhabung und zeigt einen konstruktionsmethodischen Ansatz zur Bewertung systemtechnischer Lösungsansätze mit zugehörigerer Sensitivitätsanalyse auf.
\end{abstract}

\section{Einleitung}

Beutelverpackungen werden in den Branchen der Nahrungsmittel- und Kosmetikindustrie sowie der Elektronikbranche und in zahlreichen Zweigen des Anlagen- und Maschinenbau verwendet. In vielen Fällen ist es üblich, Stückgüter kleinerer Abmessungen und gleicher Art zum Fördern und Zwischenlagern in einer abgezählten oder abgewogenen Menge in Kunststofffolienbeutel, auf denen hier in diesem Artikel der Fokus liegen soll, abzupacken. Beispiele sind Verbindungselemente wie Rohrfittings in der wasserführenden Haushaltstechnik oder Verschraubungsstücke und Steckverbindungen für pneumatische oder hydraulische Anlagen. Wenn diese Artikel gleichzeitig häufig in größeren, sehr unterschiedlichen Stückzahlen geordert werden, können diese nicht ohne weiteres zusätzlich in fixer, abgezählter Menge in Kartons verpackt werden, sondern müssen in Beuteln gehandhabt werden. Auch wird der Beutel aufgrund seiner Vorteile nur ungern durch eine leichter zu handhabende Verpackung, wie z. B. den Karton, ersetzt. Diese Vorteile sind u. a. kostengünstige Herstell- und Entsorgungskosten, gute Volumennutzung, geringes Gewicht, Flexibilität der Verpackungsgestalt und -größe bei der Herstellung, flüssigkeits- und luftdichte Verpackung, Widerstandsfähigkeit gegen Chemikalien, etc.

Häufig lastet ein hoher Kostendruck auf dem Bereich der Kommissionierung, gleichzeitig existiert dort aber auch ein großes Rationalisierungspotential. Die Automatisierung ist ein attraktives Werkzeug, um Kommissionierfunktionen effizienter zu gestalten. Bei Beutel-Artikeln gestaltet sich aus offensichtlichen Gründen jedoch eine Automatisierung der Handhabungsvorgänge nicht einfach:

Aufgrund der unvorhersehbaren Gestalt und Lage der zu handhabenden Objekte ergeben sich Schwierigkeiten bei der Bilderfassung, welche ein gezieltes automatisiertes Greifen sowie andere automatische Handhabungsvorgänge behindern. Innerhalb erforderlicher Toleranzen ist eine Positions- oder Gestaltbestimmung nicht mit ausreichender Geschwindigkeit möglich, da zum Teil sehr hohe Taktraten erreicht werden müssen. Darüber hinaus werden durch die unvorhersehbare Reaktion der Objekte auf Kräfte die Einsatzmöglichkeiten von Greifern eingeschränkt. Hinzu kommt, dass das verpackte Gut häufig in loser Schüttung in Gitterboxen oder anderen Behältnissen gelagert und im Kommissionierbereich bereitgestellt wird, wodurch der Automatisierer zunächst mit der hinlänglich bekannten und selten mit gerechtfertigtem Aufwand gelösten Problematik des „Griffs in die Kiste“ konfrontiert wird. Zurzeit wird entsprechende Ware deshalb ausschließlich manuell mit hohem Personalaufwand kommissioniert. 
Beutel sind biegeweiche Objekte, d. h., sie verformen sich unter Einfluss von Torsions- und Biegemomenten bzw. Druckkräften relativ stark, während sie Zugkräfte verhältnismäßig gut aufnehmen können [Böger97]). Aus der Literatur ist allgemein bekannt, dass biegeweiche Teile (Werkstücke) bei der Automatisierung von Handhabungsvorgängen Probleme bereiten [Hesse96]. Bei befüllten Beuteln ist zusätzlich der Einfluss durch das verpackte Gut zu beachten. Hier gibt es einige ungewöhnliche Ansätze. So ist von Turner et al. [Turner94] bekannt, dass sie versucht haben, vollautomatisch Beutel auf einem durch speziell angeordnete Düsen (air-jets) erzeugten Luftbett zu fördern, sie zu orientieren und in einen Gurt einzuhängen, um sie zu magazinieren. Diese aufwendige Anlage wurde jedoch nicht über das Versuchsstadium hinaus weiterentwickelt. Hingegen bieten einige Verpackungsmaschinenhersteller heutzutage zu ihren Produkten automatische Handhabungseinrichtungen an, welche direkt nach dem Eintüten von Artikeln die befüllten Beutel abtransportieren und in einer abgezählten Menge Kartons oder einem Kartonierprozess zuführen.

Da die Beutel nach dem Verpackungsprozess in vereinzelter Form vorliegen, sind Handhabungsoperationen noch verhältnismäßig einfach. Die eigentliche Schwierigkeit bei der automatischen Beutelhandhabung besteht in der Vereinzelung von Beuteln aus einem Haufwerk oder losen Schüttung heraus, wie man sie in einem Kommissionierlager vorfindet. In der Literatur sind daher kaum Versuche dokumentiert, die explizit zum Ziel haben, eine automatische Handhabung bei der Kommissionierung von Beuteln in den Griff zu bekommen.

Die nachfolgenden Beschreibungen sollen zunächst die Randbedingungen für eine automatische Handhabung zum Kommissionieren von Beuteln durch Produkt- und Verpackungseigenschaften aufzeigen. Da dabei nicht auf jede Beutelverpackung im Einzelnen eingegangen werden kann, werden spezielle Beispiele aufgegriffen. Bei der Entwicklung automatischer Anlagen ist es erforderlich, systematisch vorzugehen. Es werden daher im Anschluss konstruktionsmethodische Hinweise zur Annäherung an die schwierige Aufgabe der Entwicklung eines automatisierten Systems zur Handhabung von Beuteln gegeben.

\section{Beeinflussbare Größen}

Im Folgenden soll ein Überblick über die Herstellung der Verpackung gegeben sowie auf die physikalischen und lagertechnischen Eigenschaften der Produkte eingegangen werden.

\section{Herstellung der Beutelverpackung und Beutelformen}

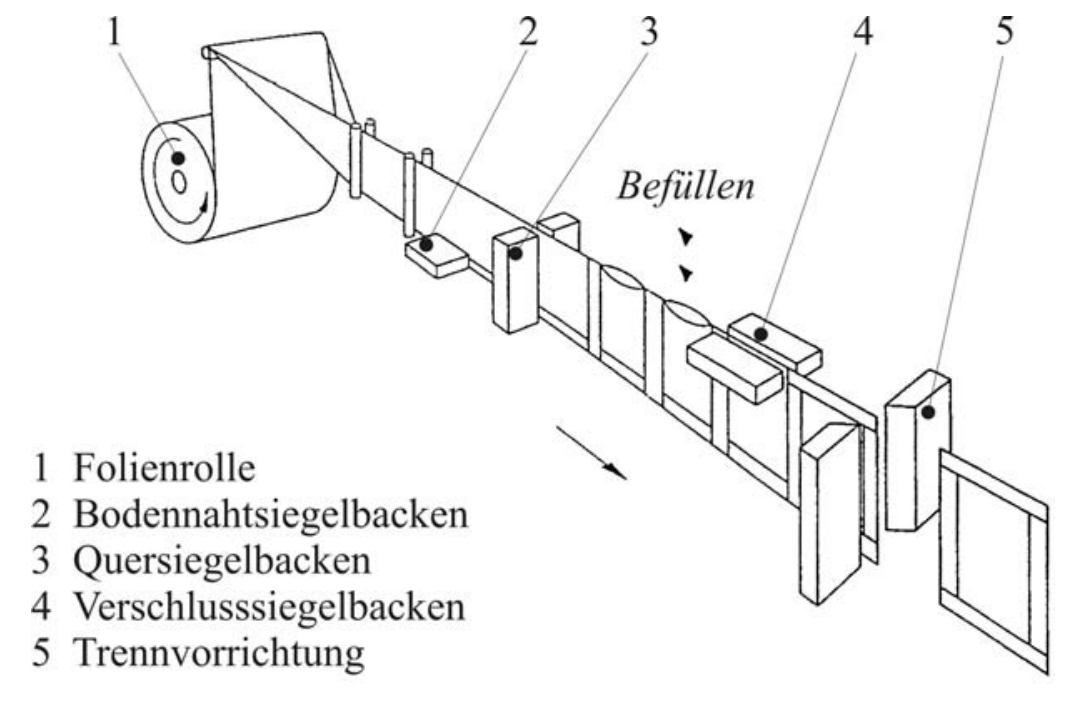

Abb. 1: Horizontal-Form-, Füll- und Verschließmaschine [DIN EN 415-3] 


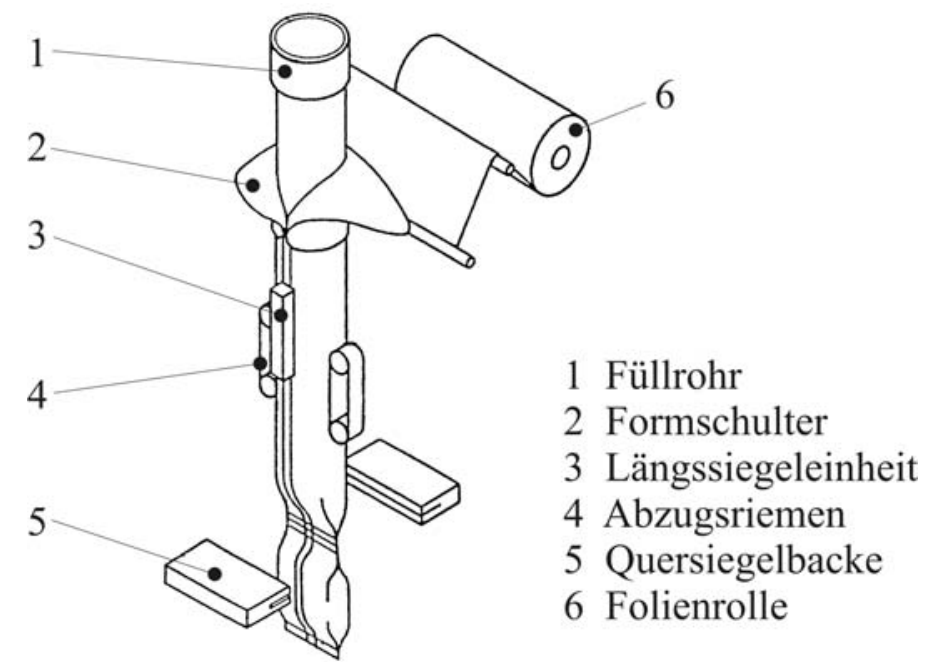

Abb. 2: Vertikal-Form-, Füll- und Verschließmaschine [DIN EN 415-3]

Um einen Eindruck über die Möglichkeiten der handhabungs- und automatisierungsgerechten Gestaltung von Beuteln zu bekommen, sollte zunächst der Herstellprozess dieser Verpackung genauer untersucht werden. Da es unmöglich ist, die Funktionsweise aller Verpackungsmaschinen und deren Varianten an dieser Stelle aufzulisten und zu erläutern, sollen stellvertretend zwei der am häufigsten angewendeten Verfahren zur Herstellung von Kunststofffolienbeuteln für Stückund Schüttgüter hervorgehoben werden.

Abbildung 1 verdeutlicht das Verfahren der Horizontal-Form-, Füll- und Verschließmaschine. Beim horizontalen Befüllen wird zu Beginn die von einer Rolle abgezogene Folienbahn gefaltet. Je nach Bedarf kann im Anschluss der Boden heißgesiegelt ${ }^{1}$ oder verschweißt ${ }^{2}$ werden. Danach wird die Bahn quer zur Vorschubrichtung heißgesiegelt. Zwischen den Quersiegelnähten wird der Strang zur Befüllung geöffnet. Abschließend wird die befüllte Kammer verschlossen und ein Beutel vom Strang abgetrennt. Beim vertikalen Befüllen (vgl. Abbildung 2) wird die Folie anfangs über eine Formschulter gezogen, wodurch sie sich schlauchförmig um das Füllrohr legt, und in Längsrichtung heißgesiegelt. Nach Einfüllen des Gutes verschließen Quersiegelbacken den Beutel, wobei meistens ein mit der Backenbewegung mitgeführtes Messer den befüllten Beutel gleichzeitig abtrennt.
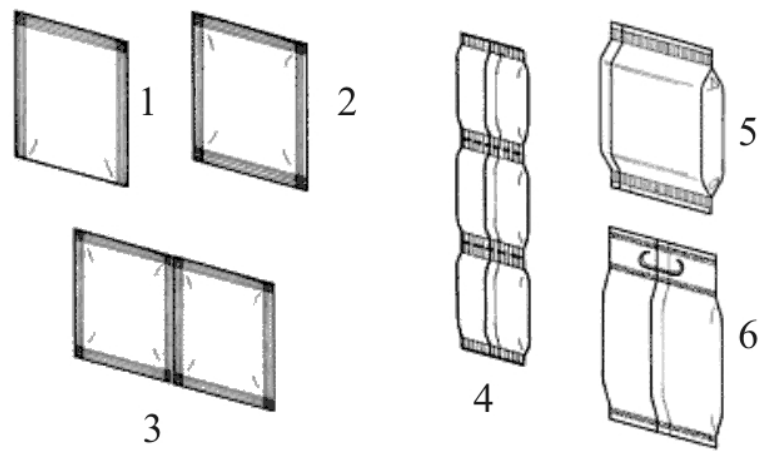

\section{3-Seiten-Siegelrandbeutel \\ 2 4-Seiten-Siegelrandbeutel \\ 3 Doppel-Siegelrandbeutel \\ 4 Kettenbeutel \\ 5 Seitenfaltbeutel \\ 6 Tragegriffbeutel}

Abb 3: Herstellbare Flachbeutelformen [Quelle: http://www.rovema.de - Stand: 03/2005]

Als Folienmaterial wird in den meisten Fällen Polyethylen (PE), Polypropylen (PP) oder Polyethylenterephthalat (PET) verwendet [Ahlhaus97]. Die Folien können dabei eine oder mehrere unterschiedlich geartete Schichten (Verbundfolien) aufweisen, die bestimmte Funktionen übernehmen (Bedruckbarkeit, Heißsiegelbarkeit etc.). Abbildung 3 zeigt einige der mit den

${ }^{1}$ Heißsiegeln ist ein Verfahren zum Verbinden thermoplastischer Schmelzschichten bei Packstoffen durch Heißpressen und wird dort eingesetzt, wo ein Verschweißen aufgrund ungünstiger Stoffpaarungen nicht möglich ist (vgl. [NN99]).

${ }^{2}$ Im Folgenden soll stellvertretend für beide Verfahren nur der Begriff Heißsiegeln verwendet werden. 
beschriebenen Verfahren herstellbaren Flachbeutelformen ${ }^{3}$. Mit etwas zusätzlichem Aufwand sind Bodenteile in den Beutel einbringbar, so dass mit diesen Verfahren auch standfähige Beutel herstellbar sind (Abbildung 4). Diese Beutelform ist für eine Handhabung vorteilhaft, da sie beim Aufrechterhalten von Ordnungszuständen hilfreich ist. Jedoch leuchtet ein, dass dadurch Kosten zunehmen, Taktzeiten erhöht werden und die erhöhte Steifigkeit der Verpackung dazu führt, dass in loser Schüttung mehr Volumen für die gleiche Anzahl Artikel eingenommen wird.

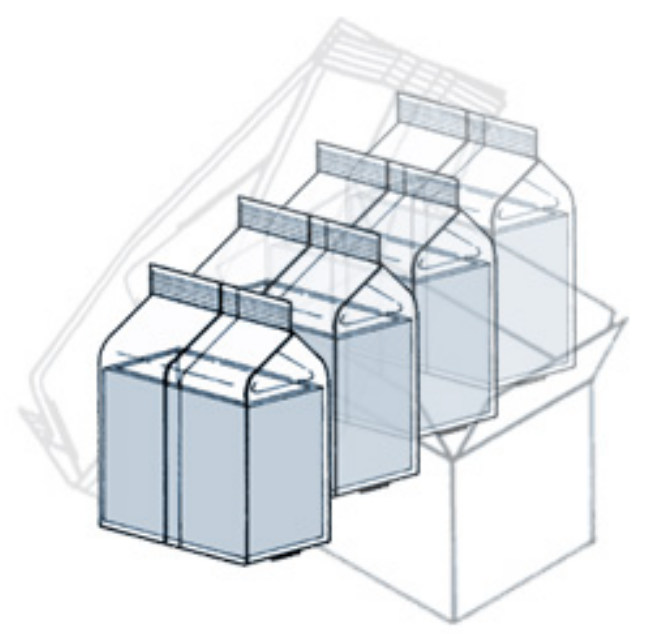

Abb. 4: Standfähige Blockbodenbeutel [Quelle: http://www.rovema.de - Stand 03/2005]

Neben der Erzeugung von Bodenteilen bieten sich weitere Gestaltungsmöglichkeiten. Jede Maßnahme, die das oberste Automatisierungsgebot „Ein einmal hergestellter Ordnungszustand ist aufrecht zu erhalten“ unterstützt, ist zu begrüßen. Einflüsse, die mit verhältnismäßig geringem Änderungsaufwand auf die Form genommen werden können, sind:

- Beutellänge und -breite

- Breite der Siegelnähte

- Lochungen in den Siegelnähten

- Beutelfüllgrad

- Verkettung von Beuteln.

Ein Erhöhen des Beutelfüllgrades kommt einer Versteifung des Beutels gleich. Es ist leicht vorzustellen, dass ein prall gefüllter Beutel sich weniger gut mit anderen Beuteln verhaken kann wie ein nur gering befüllter Beutel. Eine Verkettung von Beuteln ist in Abbildung 3 durch Darstellung 4 verdeutlicht. Im Normalfall ist die Zahl der auf diese Weise verkettbare Beutel nicht begrenzt.

Neben der Form sind auch einige optische Eigenschaften und Materialeigenschaften beeinflussbar:

- Lichtdurchlässigkeit

- Aufdruck

- Einsatz von Schrumpffolie

- $\quad$ Foliendicke (Auswirkung auf Steifigkeit und Festigkeit)

Zur Verbesserung automatischer Erkennung (Farbsensoren, Lichtschranken, usw.) kann es von Vorteil sein, die Folie auf eine bestimmte Art zu färben oder zu bedrucken oder eine bestimmte Lichtdurchlässigkeit einzustellen. Diese Attribute sind ohne eine Änderung des Verpackungsprozesses zu erreichen, es muss nur der Packstoff ausgetauscht werden. Die übrigen Eigenschaften können Auswirkungen auf den Herstellprozess haben. Maschinenparameter sind gegebenenfalls entsprechend abzuändern (z. B. Siegelzeit bei Änderung der Foliendicke) bzw. zusätzliche maschinelle Anforderungen zu erfüllen (Heißgebläse bei Einsatz von Schrumpffolie). In begrenztem Maße sind auch Reibkoeffizienten beeinflussbar.

Es ist darauf zu achten, dass Vor- und Nachteile der Änderungen im Vorfeld gut abgewogen werden. Eine Erhöhung des Beutelfüllgrades kann beispielsweise zur Verbesserung von Handhabungsaufgaben beitragen, auf der anderen Seite werden die Teile u. U. daran gehindert, sich in einer losen Schüttung raumsparend anzuordnen.

${ }^{3}$ Flachbeutel sind Beutel ohne konstruktives Bodenteil [Bleisch03]. 


\section{Operationen im Lager}

Um den Kommissionierprozess besser zu verstehen, sollen mit den standardisierten Symbolen der VDI-Richtlinie 2860 [VDI90] zunächst Funktionsstrukturen des übergeordneten Materialflusses und des Kommissioniervorgangs verdeutlicht werden. Abbildung 5 zeigt eine Möglichkeit der Abstraktion der Materialflussvorgänge von der Zuführung der Artikel zum Verpackungsprozess bis zum Kommissionieren, welches durch die Funktion Zuteilen repräsentiert wird. Je nach Strategie können die Funktionen Fördern oder eine Zwischenlagerung entfallen. Der Kommissioniervorgang vom Erkennen der richtigen Artikel bis zu deren Sammlung im Kommissionierbehälter lässt sich nun detaillierter, wie in gezeigt, darstellen. Auch hier ist der Wegfall einiger Funktionen denkbar. Nach den Regeln der Konstruktionssystematik kann aus diesen Funktionsstrukturen ein morphologischer Kasten zur Entwicklung von Lösungsvarianten erstellt werden. Der morphologische Kasten ist in der Literatur ausreichend beschrieben (z.B. [Pahl04]), so dass an dieser Stelle nicht näher darauf eingegangen werden soll.

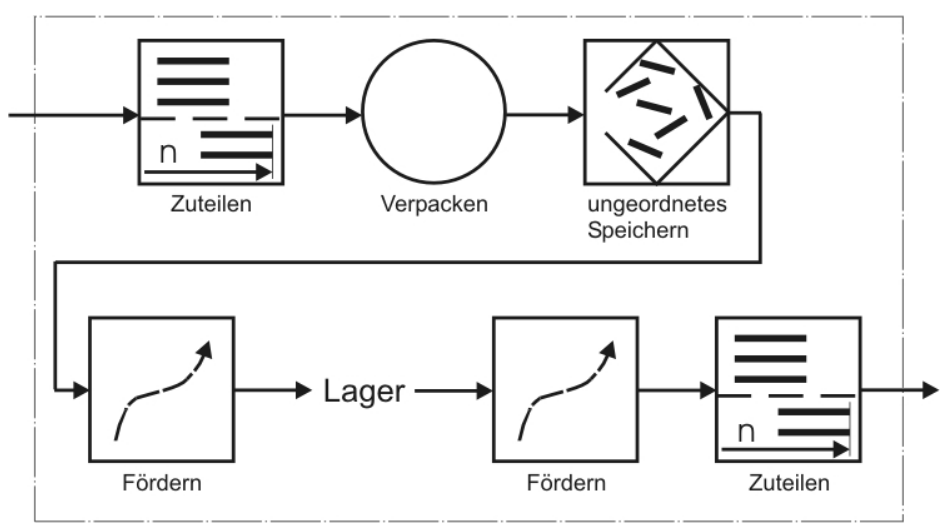

Abb. 5: Übergeordneter Materialfluss

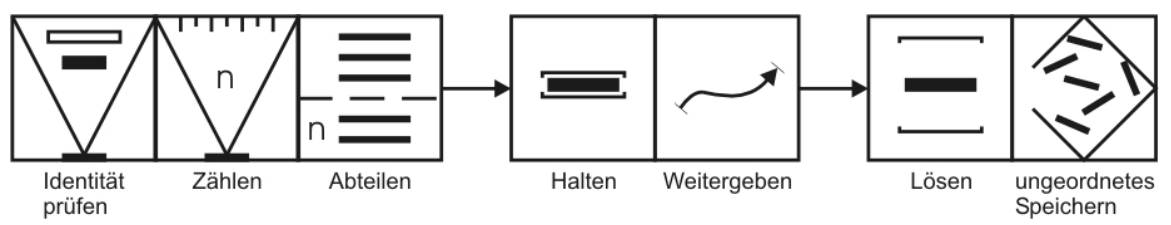

Abb. 6: Der Kommissioniervorgang

Es ist also die Bedingung, dass zu gestaltende Förder- und Handhabungseinrichtungen den aufgestellten Funktionsstrukturen und einem effizienten Materialfluss gerecht werden. Die Entwicklung eines Teilsystems hat Auswirkung auf den gesamten Materialfluss und muss somit auf eine Optimierung des gesamten Systems abzielen. So rücken zusätzlich geeignete Ladehilfsmittel (LHM) ins Auge des Entwicklers, aber auch die Eigenschaften der Produkte und ihrer Verpackung bestimmen maßgeblich die Möglichkeiten der Anlagengestaltung. Weiterhin sind nicht nur die geometrische Form und physikalische Eigenschaften der Produkte zu beachten, sondern für die Gestaltung von Handhabungsgeräten sind insbesondere die zu erreichenden Taktzahlen und statistische bzw. probabilistische Daten über die Anzahl der Beutel eines bestimmten Artikels pro Auftrag von Interesse. Solche Daten bestimmen beispielsweise, welche Antriebs- und Steuerungstechnologien einsetzbar sind und wie der Vereinzelungsvorgang abzulaufen hat (ob z. B. mehrere Beutel gleichzeitig abteilbar sein sollten o. ä.). 


\section{Methodische Vorgehensweise bei der Gestaltung}

\section{Problematik der Wirtschaftlichkeitsbeurteilung}

Unerlässlich vor der Einführung, aber nicht ohne weiteres durchführbar, sind Abschätzungen zur Wirksamkeit von Maßnahmen zur Rationalisierung von Kommissionierbereichen. Mit Methoden der Kostenrechnung wie Investitionsrechenverfahren lassen sich nur monetarisierbare Eigenschaften bewerten. Den auf diese Weise messbaren Veränderungen stehen Effekte gegenüber, die in ihrer Wirkung nur schwer zu quantifizieren sind [Becker93], wie z. B. der Nutzen einer ergonomischen Anlagengestaltung oder der Imagegewinn durch Einsatz moderner Technologien (was häufig bei der Einführung von Robotersystemen angeführt wird). Weiterhin stehen vor einer Verwirklichung zu einem Großteil noch nicht quantifizierbare Beurteilungsmerkmale zur Verfügung.

Für eine sinnvolle Gesamtbeurteilung ist die Menge aus strategischen, technischen, wirtschaftlichen und sozialen Aspekten zu berücksichtigen, zwischen denen komplexe Interdependenzen vorherrschen können. Damit diese Einflüsse dennoch Berücksichtigung finden, bietet sich eine Bewertung mit an die Methode der Nutzwertanalyse (NWA) [Zangemeister76] angelehnten Verfahren an. Diese Verfahren stellen in Kombination mit den Kostenrechnungsverfahren ein brauchbares Werkzeug zur Entscheidungsfindung dar. Sie bieten die Möglichkeit, auch qualitative oder aufgrund eines frühen Entwicklungsstandes noch nicht quantifizierbare Eigenschaften und Effekte von Maßnahmen in die Beurteilung einfließen zu lassen.

Für eine sinnvolle Gesamtbeurteilung ist die Menge aus strategischen, technischen, wirtschaftlichen und sozialen Aspekten zu berücksichtigen, zwischen denen komplexe Interdependenzen vorherrschen können. Damit diese Einflüsse dennoch Berücksichtigung finden, bietet sich eine Bewertung mit an die Methode der Nutzwertanalyse (NWA) von Zangemeister [Zangemeister76] angelehnten Verfahren an. Diese Verfahren stellen in Kombination mit den Kostenrechnungsverfahren ein brauchbares Werkzeug zur Entscheidungsfindung dar. Sie bieten die Möglichkeit, auch qualitative oder aufgrund eines frühen Entwicklungsstandes noch nicht quantifizierbare Eigenschaften und Effekte von Maßnahmen in die Beurteilung einfließen zu lassen. Die Anwendung von NWA-ähnlichen Methoden ist heute weit verbreitet und immer noch höchst aktuell. Das Anwendungsfeld reicht vom Bereich der Investitionsentscheidungen für Produktionssysteme [Köhrmann97] bis zum Einkommenssteuerrecht [Schmidt02], der Bewertung von Software-Systemen [Algedri98] und zur Projektbewertung [Schulte02].

\section{Bewertung von Varianten}

Eine Abwandlung der NWA, um Lösungsvarianten, seien es Konzepte oder bereits detailliertere Entwürfe, zu bewerten, stellt sich wie folgt dar: Es geschieht eine Aufstellung von $K$ relevanten Zielkriterien, welche möglichst unabhängig voneinander sein sollten. Diese werden eingeteilt in $T$ technische und $K-T$ wirtschaftliche Kriterien und relativ zueinander gewichtet, so dass für die Gewichtungen $g_{m}^{t}$ und $g_{m}^{w}$ der technischen und wirtschaftlichen Kriterien gilt:

$$
\begin{aligned}
& \sum_{m=1}^{T} g_{m}^{t}=\sum_{m=T+1}^{K} g_{m}^{w}=1, \\
& g_{m}^{t}, g_{m}^{w} \geq 0 .
\end{aligned}
$$

Mit den zusätzlichen Gewichtungsfaktoren $c^{t} \geq 0$ und $c^{w} \geq 0$ für die technischen und die wirtschaftlichen Kriterien, für die $c^{t}+c^{w}=1$ gilt, kann somit definiert werden: 


$$
\begin{aligned}
& g_{m}=\left\{\begin{array}{l}
c^{t} \cdot g_{m}^{t}, m \leq T \\
c^{w} \cdot g_{m}^{w}, m>T
\end{array}\right. \\
& \sum_{m=1}^{K} g_{m}=1 .
\end{aligned}
$$

Jede Variante $n$ wird hinsichtlich eines Kriteriums $m$ mit einer Zahl (Zielwert) $0 \leq w_{m n} \leq 10$ bewertet. Je nach Kriterium werden unterschiedliche Methoden oder Wertfunktionen verwendet, mit denen versucht wird, Objektivität in die Bewertung einfließen zu lassen. Aus den Bewertungen werden die technische $W^{t}$, die wirtschaftliche $W^{w}$ und die gesamte gewichtete Wertigkeit $W$ je Variante $n$ bestimmt:

Es wird zunächst angenommen, dass technische und wirtschaftliche Wertigkeit gleichgewichtig sind und somit $W_{n}=\left(W_{n}^{t}+W_{n}^{w}\right) / 2$ gilt.

$$
\begin{aligned}
& W_{n}^{t}=\sum_{m=1}^{T} g_{m}^{t} \cdot w_{m n} \\
& W_{n}^{w}=\sum_{m=T+1}^{K} g_{m}^{w} \cdot w_{m n} \\
& W_{n}=\sum_{m=1}^{K} g_{m} \cdot w_{m n} .
\end{aligned}
$$


Tabelle 1: Bewertungstabelle

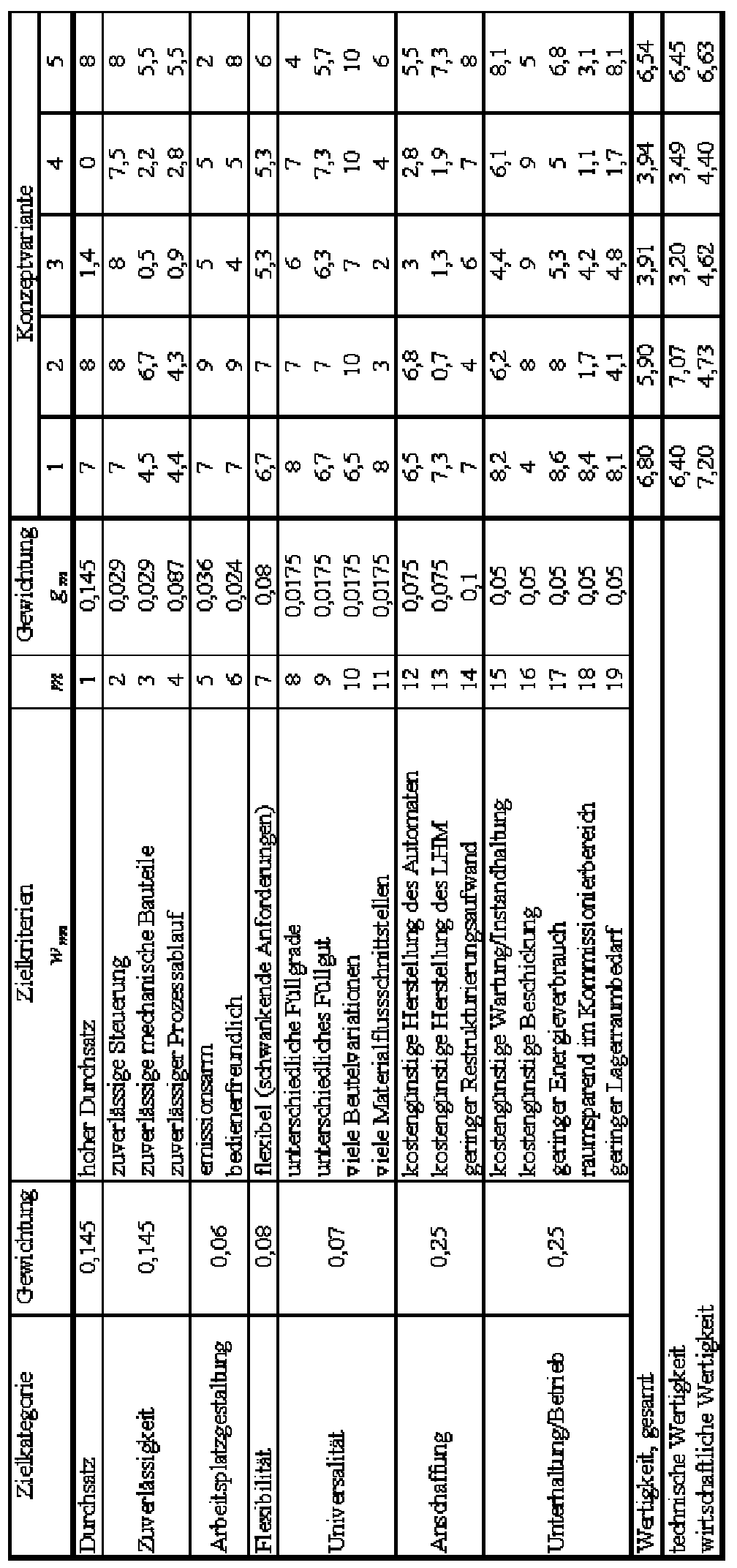


In Anlehnung an eine Auflistung für Entscheidungskriterien für Kommissioniersysteme aus der VDI-Richtlinie 3590 [VDI02] sind die nachfolgenden technischen Zielkategorien für Zielkriterien hergeleitet worden:

- Durchsatz

- Zuverlässigkeit

- Arbeitsplatzgestaltung

- Flexibilität

- Universalität.

Durch Aufspaltung dieser Kategorien ergeben sich Zielkriterien. Beispielsweise lassen sich für die Kategorie Zuverlässigkeit die Kriterien zuverlässige Sensorik, zuverlässige Mechanik und zuverlässiger Prozessablauf finden. Kategorien für wirtschaftliche Kriterien lassen sich wie folgt darstellen:

- Investitionen

- Unterhaltung/Betrieb

Je nach Detaillierungsgrad der Varianten lässt sich auch noch die Kategorie Entsorgung zur Bewertung hinzunehmen, so dass der gesamte Lebenszyklus einer technischen Anlage abgedeckt ist. Tabelle 1 zeigt beispielhaft eine solche Bewertung von fünf Varianten eines Kommissioniersystems (auf die hier nicht näher eingegangen werden soll).

\section{Sensitivitätsanalyse}

Das beschriebene Bewertungsverfahren ist leider je nach Detaillierungsgrad mit Unsicherheiten verbunden, da Werte sowohl für $g_{m}$ als auch $w_{m n}$ geschätzt werden müssen. Je weiter die Entwicklung der Varianten vorangeschritten ist, desto exakter lassen sich die Eigenschaften dieser hinsichtlich der Zielkriterien abschätzen. In der Regel bleibt aber eine gewisse Restunsicherheit, die die Stabilität des Ergebnisses der Bewertung in Frage stellt. Um diese Stabilität zu kontrollieren, sollten Sensitivitätsanalysen ${ }^{4}$ durchgeführt werden.

\section{Theorie der Analysemethode}

Es wird an dieser Stelle eine in der Literatur bisher nicht dokumentierte Vorgehensweise hergeleitet und vorgeschlagen: Die Ermittlung der geeignetsten Variante nach obigem Vorgehen kann mathematisch als Optimierungsprogramm beschrieben werden. Falls die geeignete Variante $n^{*}$ beispielsweise als diejenige mit der höchsten Gesamtwertigkeit $W_{n^{*}}=\max _{n} W_{n}$ definiert ist, lässt sich schreiben:

$$
\begin{aligned}
& \max \sum_{n} W_{n} x_{n} \\
& \text { mit } \sum_{n} x_{n}=1 \\
& \quad x_{n} \in\{0,1\} \forall n,
\end{aligned}
$$

wobei alle $x_{n}$ binäre Entscheidungsvariablen und alle anderen Größen durch die vorangegangene Bewertung festgelegte Koeffizienten sind. Die Aufstellung eines Programms für die technische oder wirtschaftliche Wertigkeit geschieht analog. Die Koeffizienten dieses Programms sind subjektiv über die Werte $g_{m}$ und $w_{m n}$ festgelegt worden und somit nicht gesichert. Um Aussagen über die Stabilität der Lösung treffen zu können, ist zu ermitteln, welche dieser Werte wie stark geändert werden müssen, bis eine Änderung der Lösung eintritt, und zu überprüfen, wie realistisch diese Änderungen sind.

Die Änderung der Gewichtungen $g_{m}$ wird mit $d_{m}$ bezeichnet. Da $\sum_{m=1}^{K} g_{m}=1$ (s. o.) weiterhin gelten muss, werden die neuen Gewichtungen $g_{m}$ ' beschrieben durch:

$$
g_{m}^{\prime}=\frac{g_{m}+d_{m}}{1+\sum_{m} d_{m}}
$$

\footnotetext{
${ }^{4}$ in der Literatur stellenweise auch als Sensibilitäts- oder Empfindlichkeitsanalysen bezeichnet
} 
Der neue Gesamtwert einer Variante $n$ ergibt sich mit $w_{m n}=$ const somit zu:

$$
W_{n}{ }^{\prime}=\sum_{m} g_{m}{ }^{\prime} w_{m n}=\frac{1}{1+\sum_{m} d_{m}} \sum_{m}\left(g_{m}+d_{m}\right) \cdot w_{m n} \text {. }
$$

Es sei angenommen, dass $d_{m}=d \neq 0$ jeweils nur für ein bestimmtes $m$ und ansonsten $d_{m}=0$ gilt, so dass $\sum_{m} d_{m} \stackrel{m}{=} d$. Dann kann die Änderung gegenüber der alten Wertigkeit $\delta_{m n}^{m}=W_{n}{ }^{\prime}-W_{n}$ unter Verwendung der Definition von $W_{n}$ (s. o.) formuliert werden als

$$
\delta_{m n}=\frac{d}{1+d} \cdot\left(w_{m n}-W_{n}\right) \text {. }
$$

Eine Änderung der optimalen Lösung tritt nun im Fall

$$
\delta_{m n}^{*}+W_{n} \geq \delta_{m n^{*}}^{*}+W_{n^{*}}
$$

für irgendein $n \neq n^{*}$ ein. Einsetzen von $\delta_{m n}$ ergibt

$$
d_{m n}^{*} \leq \frac{W_{n}-W_{n^{*}}}{w_{m n^{*}}-w_{m n}}
$$

Einsetzen in obige Gleichung für $g_{m}{ }^{\prime}$ ergibt $g_{m n}^{*}$, und somit folgt für die Änderung der Gewichtung $\Delta g_{m n}^{*}=g_{m n}^{*}-g_{m}$

$$
\Delta g_{m n}^{*} \geq \frac{d_{m n}^{*}}{1+d_{m n}^{*}}\left(1-g_{m}\right)
$$

Diese Änderung einer bestimmten Gewichtung eines Kriteriums $m$ bedingt auch zwangsläufig Änderungen in den übrigen Gewichtungen, für die $d_{m}=0$ gehalten wurde (s. Gleichung (5)). Alle auf diese Weise erzeugten Änderungen müssen gültig sein, was heißt, dass die Bedingungen aus Gleichung (1) und (2) erfüllt bleiben müssen, also u. a. gilt

$$
0 \leq g_{m}+\Delta g_{m n}^{*} \leq 1 \text {. }
$$

Damit ist ein erstes Werkzeug für die Analyse der Koeffizienten $g_{m}$ des Optimierungsproblems hergeleitet. Mit einfachen Tabellenberechnungen in z. B. MS-Excel ${ }^{\circledR}$ lassen sich zunächst für alle $n \neq n^{*}$ und alle $m$ die Werte $d_{* m n}^{*}$ und daraus anschließend alle $\Delta g_{m n}^{*}$ bestimmen. Hat diese Analyse sehr niedrige Werte $\Delta g_{m n}^{* m n}$ zur Folge, ist die Lösung des Optimierungsprogramms u. U. als labil zu erachten.

Des Weiteren beeinflussen die Werte $w_{m n}$ die Koeffizienten $W_{n}$ des Optimierungsprogramms. Es sei angenommen, dass wiederum jeweils nur ein Wert $w_{m n}$ mit $n \neq n$ variiert wird, während alle anderen $w_{m n}$ konstant gehalten werden. Die Änderung von $W_{n}$ sei mit $\Delta w_{m n}$ bezeichnet. Damit die optimale Lösung $n^{*}$ sich ändert, muss dann gelten:

$$
\Delta w_{m n}^{*}=\frac{W_{n^{*}}-W_{n}}{g_{m n}} .
$$

Darunter sind diejenigen Änderungen gültig, die die Bedingung

$$
w_{m n}+\Delta w_{m n}^{*} \leq 10
$$

erfüllen. Umgekehrt ist es auch möglich, $w_{m n^{*}}$ um $\Delta w_{m n}^{*}$ zu erniedrigen, um die Lösung von obigem Optimierungsprogramm zu verändern, wobei

$$
0 \leq w_{m n^{*}}-\Delta w_{m n}^{*}
$$


gelten muss. Ein weiteres Werkzeug besteht also darin, über einfache Tabellenberechnung für alle $n \neq n^{*}$ und alle $m$ die Werte $\Delta w_{m n}^{*}$ zu bestimmen und zu ermitteln, ob sich so niedrige, gültige Werte ergeben, dass die ursprüngliche Lösung als labil zu erachten ist.

Darüber hinaus werden die Werte $\Delta w_{m n}$ am geringsten ausfallen, welche die größten zugehörigen $g_{m}$ besitzen, da für die Änderung der Wertigkeit gilt:

$$
\delta_{m n}=\sum_{m} g_{m} \Delta w_{m n}^{*}
$$

Daraus lässt sich eine weitere Methode ableiten: Die Werte $g_{m}$ werden für alle $m$ der Größe nach sortiert und je Variante $n \neq n^{*}$ entsprechend dieser Folge (angefangen mit dem Größten) die zugehörigen Werte $\Delta w_{m n}^{*}$ der Reihe nach um einen festzulegenden zulässigen Höchstfaktor geändert, bis sich die Lösung $n^{*}$ ändert. Die Anzahl der Werte, welche erhöht werden müssen, bis eine Änderung eintritt, kann als Kriterium für die Stabilität der Lösung verwendet werden.

\section{Anwendung der Methode}

Die im Abschnitt zuvor dargestellte Methode wird zur Verdeutlichung auf die Bewertung aus Tabelle 1 angewendet. Die Analyse der Sensitivität des Optimums $n^{*}=1$ für die Gesamtwertigkeit hinsichtlich Änderungen der Gewichtungen $g_{m}$ ergab Tabelle 2, eine Analyse des Optimums $n_{t}^{*}=2$ für die technische Wertigkeit. Eine Analyse des wirtschaftlichen Optimums erübrigt sich, da $n^{*}=n_{w}^{*}$ und $W_{n^{*}}-W_{n}<W_{n^{*}}^{w}-W_{n}^{w} \forall n \neq n^{*}$ und somit $\Delta g_{m n^{*}} \leq \Delta g_{m n^{*}}^{w}$. In den Tabellen enthalten einige Zellen keinen Eintrag, da $w_{m n^{*}}-w_{m n}=0$ und somit keine Änderung der optimalen Lösung möglich ist. Weiterhin sind auch als ungültig definierte Werte aufgeführt.

Tabelle 2: Zum Wechsel der gesamt gesehen optimalen Lösung $n^{*}=1$ erforderliche Gewichtungsänderungen $\Delta g_{m n}^{*}$ der Kriterien $g_{m}$

\begin{tabular}{|c|cccc|}
\hline Kriterium & \multicolumn{4}{|c|}{$\Delta g_{m}^{*}$ der Variante $n$} \\
& 2 & 3 & 4 & 5 \\
\hline 1 & 0,406 & $-0,912$ & $-0,589$ & 0,176 \\
2 & 0,461 & 0,721 & 0,826 & 0,200 \\
3 & 0,283 & $-2,529$ & 4,983 & 0,200 \\
4 & 1,027 & $-4,328$ & 2,075 & 0,174 \\
5 & 0,300 & 3,130 & 3,215 & $-0,053$ \\
6 & 0,304 & $-25,703$ & 3,255 & 0,201 \\
7 & 0,691 & 1,784 & 1,804 & $-0,542$ \\
8 & $-9,136$ & 3,190 & 1,512 & $-0,068$ \\
9 & 0,737 & 1,140 & 0,812 & $-0,344$ \\
10 & 0,201 & 0,838 & 0,442 & $\mathbf{0 , 0 6 8}$ \\
11 & $-0,217$ & $-0,913$ & $-2,455$ & $-0,147$ \\
12 & 0,694 & $-4,385$ & $-3,133$ & $-0,324$ \\
13 & $-0,147$ & $-0,860$ & $-1,039$ & - \\
14 & $-0,387$ & 1,376 & - & 0,185 \\
15 & $-0,782$ & $-3,018$ & 3,587 & 1,545 \\
16 & 0,175 & 0,348 & 0,345 & 0,196 \\
17 & 2,832 & $-6,701$ & $-3,651$ & $-0,160$ \\
18 & $-0,148$ & $-2,096$ & $-0,611$ & $\mathbf{- 0 , 0 4 9}$ \\
19 & $-0,277$ & $-6,701$ & $-0,766$ & - \\
\hline
\end{tabular}


Es wird festgelegt, dass gültige Werte, deren Betrag kleiner als 0,1 ist, als kritisch anzusehen sind und eine ausreichende Stabilität des Ergebnisses der Bewertung in Tabelle 1 nicht gegeben ist. Bei vergleichender Betrachtung mitTabelle 1 ergeben sich zwei kritische Werte in Tabelle 2. Diese Werte sind fett dargestellt. Das bedeutet, dass Variante 5 bei einer nur geringfügigen Erhöhung der Gewichtung des Kriteriums 10 viele Beutelvariationen oder einer geringfügigen Verringerung der Gewichtung des Kriteriums 18 raumsparend im Kommissionierbereich Variante 1 als beste Lösung ablösen könnte. Tabelle 3 enthält hingegen keine kritischen Werte, Variante 2 ist als technisch beste Lösung hinsichtlich Veränderungen der Gewichtungen relativ stabil.

Tabelle3: Zum Wechsel der technisch optimalen Lösung erforderliche Gewichtungsänderungen $\Delta g_{m n}^{t^{*}}$ der Kriterien $g_{m}^{t}$

\begin{tabular}{|c|cccc|}
\hline Kriterium & \multicolumn{4}{|c|}{$\Delta g_{m n}^{t^{*}}$ der Variante $n$} \\
& 1 & 3 & 4 & 5 \\
\hline 1 & $-1,436$ & $-1,003$ & $-0,574$ & - \\
2 & $-1,906$ & - & 1,095 & - \\
3 & $-0,412$ & $-1,559$ & $-3,653$ & $-1,001$ \\
4 & 0,719 & 6,869 & 1,422 & 0,281 \\
5 & $-0,467$ & $-26,507$ & $-7,858$ & $-0,090$ \\
6 & $-0,479$ & $-3,241$ & $-8,061$ & $-1,542$ \\
7 & 1,523 & 1,500 & 1,601 & $-1,361$ \\
8 & 0,387 & 1,302 & - & $-0,251$ \\
9 & 1,749 & 1,178 & 0,890 & $-0,875$ \\
10 & $-0,228$ & 4,313 & - & - \\
11 & 0,114 & 1,302 & 0,754 & 0,165 \\
\hline
\end{tabular}

Die Analysen im Bezug auf Änderungen der Werte $w_{m n}$ und Ermittlung der $\Delta w_{m n}^{*}$ verdeutlichen Tabelle 4 und 5. Aus ähnlichem Grund wie oben erübrigt sich eine Analyse des wirtschaftlichen Optimums. Dargestellt sind lediglich die Zeilen für Kriterien, für die wenigstens ein $\Delta w_{m n}^{*} \leq 10$. Die zugehörigen $g_{m}$ sind gleichzeitig die höchsten in einer Rangfolge der $g_{m}$. Je kleiner ein Wert $\Delta w_{m n}$ desto höher ist der Rang seiner Gewichtung $g_{m}$. Auch hier sind als ungültig definierte Werte in den Tabellen aufgeführt. Kritische Werte sind hier als gültige Werte mit Betrag kleiner als 4 festgelegt. Sechs der Werte in Tabelle 4 sind kritisch, d. h., eine kleine Erhöhung der Werte für Variante 5 hinsichtlich der Kriterien 1, 4, 7 oder 12 und/oder kleine Verringerung der entsprechenden Werte und der Werte für die Kriterien 13 und 14 für Variante 1 können eine Änderung des Ergebnisses zugunsten von Variante 5 zur Folge haben. enthält keine kritischen Werte, Variante 2 ist als technisch beste Lösung hinsichtlich Veränderungen der $w_{m n}$ relativ stabil.

Tabelle 4: Zum Wechsel der technisch optimalen Lösung $n^{t^{*}}=2$ erforderliche Änderungen

\begin{tabular}{|c|cccc|}
\hline Kriterium & \multicolumn{4}{|c|}{$\Delta w_{m n}^{*}$ der Variante $n$} \\
& 2 & 3 & 4 & 5 \\
\hline 1 & 6,227 & 19,933 & 19,701 & $\mathbf{1 , 7 9 0}$ \\
4 & 10,378 & 33,221 & 32,835 & $\mathbf{2 , 9 8 3}$ \\
7 & 11,286 & 36,128 & 35,708 & $\mathbf{3 , 2 4 4}$ \\
12 & 12,039 & 38,537 & 38,089 & $\mathbf{3 , 4 6 1}$ \\
13 & 12,039 & 38,537 & 38,089 & $\mathbf{3 , 4 6 1}$ \\
14 & 9,029 & 28,903 & 28,567 & $\mathbf{2 , 5 9 6}$ \\
\hline
\end{tabular}


Tabelle 5: Zum Wechsel der gesamt gesehen optimalen Lösung $n^{*}=1$ erforderliche Änderungen $\Delta w_{m n}^{*}$ der Bewertungen $w_{m n}$

\begin{tabular}{|c|cccc|}
\hline Kriterium & \multicolumn{4}{|c|}{$\Delta w_{m n}^{t^{*}}$ der Variante $n$} \\
& 1 & 3 & 4 & 5 \\
\hline 1 & 4,615 & 26,653 & 24,672 & 4,264 \\
4 & 7,692 & 44,422 & 41,121 & 7,107 \\
7 & 8,365 & 48,309 & 44,719 & 7,729 \\
\hline
\end{tabular}

Ein der Rangfolge der $g_{m}$ entsprechendes Erhöhen der $w_{m n}$ um einen festen Wert $\Delta w_{m n}^{*}=1$ ergab die Ergebnisse in Tabelle 5. Sie gibt Auskunft über die Anzahl von Werten $w_{m n}$, die entsprechend geändert werden müssen, um einen Wechsel der optimalen Lösung herbeizuführen. Leere Zellen bedeuten, dass ein Wechsel durch ein derartiges Vorgehen nicht möglich ist. Nur wenige Erhöhungen bei Variante 5 sind nötig, um die Varianten 1 und 2 als Optima abzulösen. Ähnliches gilt für Variante $1 \mathrm{im}$ Bezug auf Variante 2 als technisch beste Lösung. Variante 2 hingegen benötigt sehr viele Erhöhungen, um eine höhere Gesamtwertigkeit als Variante $1 \mathrm{zu}$ erlangen. Als wirtschaftlich beste Lösung kann Variante 2 die Variante 1 nicht ablösen.

Tabelle 6: Anzahl der Änderungen $\Delta w_{m n}=1$ der Bewertungen $w_{m n}$ zum Wechsel der optimalen Lösung

\begin{tabular}{|c|ccccc|}
\hline Kriterium & \multicolumn{5}{|c|}{ Variante $n$} \\
& 1 & 2 & 3 & 4 & 5 \\
\hline$w$ & 4 & - & - & - & 3 \\
$t$ & - & - & - & - & 4 \\
gesamt & - & 14 & - & - & 3 \\
\hline
\end{tabular}

\section{Fazit}

Im vorliegenden Beitrag wurde die Problematik der Automatisierung von Handhabungsvorgängen bei der Kommissionierung von in Beuteln verpackten Gütern behandelt. Hierbei wurde neben Gestaltungsmaßnahmen insbesondere eine Methodik für eine produktgerechte Entwicklung von Handhabungsgeräten aufgezeigt. Der Vorteil des entwickelten Verfahrens liegt in der einfachen Generierung der Entscheidungsparameter im Bezug auf die Stabilität einer Variantenbewertung und der Automatisierbarkeit vieler Berechnungsvorgänge (beispielsweise mittels Tabellenkalkulationsprogramm). Ein willkürliches manuelles Variieren der Bewertungsgrößen wird somit überflüssig.

Ein Vorhaben am Fraunhofer IML beschäftigt sich mit der automatischen Handhabung und Kommissionierung von in Beuteln verpacktem Gut. Derzeit werden Marktanalysen vorbereitet, die weiteres Hintergrundwissen verschaffen und $\mathrm{zu}$ einem Abgleich mit den Bedürfnissen der Industrie beitragen sollen, so dass eine bereits vorab aufgestellte All-Zweck-Anforderungsliste verifiziert und ergänzt werden kann. Diese dient als Grundlage für Systementwicklungen, bei welchen die aufgezeigte Methodik Anwendung findet und derzeit weiter verfeinert wird. Es konnten auf diese Weise bereits Entwürfe für Systemlösungen erarbeitet werden, eine Umsetzung in Prototypen ist geplant. Es sei noch betont, dass das Bewertungs- und Sensitivitätsanalyseverfahren zwar im Zusammenhang mit diesem konkreten Projekt entstanden ist, jedoch allgemein auf andere Entwicklungs- oder Planungsaufgaben vollständig übertragen werden kann. 


\section{Literatur}

[Ahlhaus97] Ahlhaus, Otto E.: Verpackung mit Kunststoffen. München: Hanser, 1997

[Algedri98] Algedri, Jamal; Frieling, Ekkehart: Beurteilung rechnergestützter FMEASysteme. In: QZ - Qualitität und Zuverlässigkeit (1998) 12, S. 1490-1495

[Becker93] Becker, Thomas: Automatische Kommissionierung - Eignungskriterien und Wirtschaftlichkeitsnachweis. Dortmund: LogBuch 1993

[Bleisch03] Bleisch, Günter (Hrsg.): Lexikon Verpackungstechnik. 1. Auflage. Heidelberg: Hüthig, 2003

[Böger97] Böger, Thomas. Beitrag zur Projektierung von Greifelementen für die Handhabung flächiger, biegeweicher Materialien. Dissertation. Dortmund: Praxiswissen Verlag 1998

[DIN00] DIN EN 415-3: Sicherheit von Verpackungsmaschinen - Teil 3: Form-, Füllund Verschließmaschinen. Berlin: Beuth Oktober 2000

[Hesse96] Hesse, Stefan: Praxiswissen Handhabungstechnik in 36 Lektionen. Renningen: Expert Verlag 1996

[Köhrmann97] Köhrmann, C.; Schimmelpfeng, Katja: Anwendung der Nutzwertanalyse im Bereich von Investitionsentscheidungen für Produktionssysteme unter besonderer Berücksichtigung der Verfügbarkeitssicherung. In: Zeitschrift für Planung \& Unternehmenssteuerung (1997) 4, S. 395-406

[NN99] ohne Verfasser: Brockhaus-Enzyklopädie - Neunter Band GOT-HERP. 19. Auflage. Mannheim: Brockhaus 1989

[Pahl04] Pahl, Gerhard; Beitz, Wolfgang: Konstruktionslehre - Methoden und Anwendung. 6. Auflage. Berlin [u.a.]: Springer 2004

[Schulte02] Schulte, Andreas; Bokermann, Ralf: Projektbewertung mit einem Ansatz der Nutzwertanalyse. Kassel: Ecovast-Verlag, 2002

[Schmidt02] Schmidt-Liebig, Axel: Gewerbebetrieb, Vermögensverwaltung und Nutzwertanalyse im Einkommenssteuerrecht. In: Betriebs-Berater (2002) 50, S. 2577-2582

[Turner94] Turner, C.; Davies, A.; Parkin, R. M.; Knight, J. A. G.: Soft Product Assembly and Manipulation. In: IEEC Computing and Control Division Colloquium on Intelligent Automation for Processing Non-Rigid Products (1994) Oktober, S. 4/1-4/5

[VDI90] VDI-Richtlinie 2860: Montage- und Handhabungstechnik Handhabungsfunktionen, Handhabungseinrichtungen; Begriffe, Definitionen, Symbole. Berlin: Beuth Mai 1990

[VDI02] VDI-Richtlinie 3590: Kommissioniersysteme - Praxisbeispiele. Berlin: Beuth August 2002

[Zangemeister76] Zangemeister, Christof: Nutzwertanalyse in der Systemtechnik. 4. Auflage. München: Wittemannsche Buchhandlung 1976 